\title{
Extracellular traps: how to isolate and quantify extracellular DNA (ET-DNA)
}

\author{
MARISKA MEIJER', MICHAE PRZEMYSEAW PRUCHNIAK', MAGDALENA ARAZNA², \\ URSZULA DEMKOW'
}

${ }^{1}$ University College Roosevelt Academy, Middelburg, The Netherlands

${ }^{2}$ Department of Laboratory Diagnostics and Clinical Immunology of Developmental Age, Warsaw Medical University, Warsaw, Poland

\begin{abstract}
When pathogens pass the physical barriers, neutrophils are the first cells that infiltrate the infected tissue. Subsequently they use several well-known strategies to destroy an invading microorganism such as phagocytosis and degranulation. Recently it was shown that neutrophils are capable of releasing their nuclear DNA in the form of an extracellular trap, know as a neutrophil extracellular traps (NETs). On the one hand, extracellular traps plays crucial role during antimicrobial defense, on the other they can cause autoimmune disorders. Therefore, rigorous quantification of ETs-DNA seems to be important both for basic research and clinical applications. This paper assesses quantification methods which were used to estimate extracellular traps formation.
\end{abstract}

Key words: DNA detection, extracellular DNA quantification, fluorescence, neutrophil extracellular trap.

(Centr Eur J Immunol 2012; 37 (4): 321-325)

\section{Introduction}

Neutrophils, also known as polymorphonuclear leukocytes (PML) are amongst the most abundant leukocytes in human beings. In adults, approximately 1 to $2 \times 10^{11}$ neutrophils are generated in the bone marrow each day [1]. They are released into the blood stream, where they circulate for four to ten hours, after which they die.

When an infective agent passes first-line defences of the body, neutrophils are the first cells that infiltrate the inflamed tissue and try to overcome the infection [2]. For this purpose, they use three main strategies. First of all, neutrophils can phagocytose invading microorganisms. A second mechanism is connected with the release of reactive oxygen species (ROS) and granular, antimicrobial proteins. Unfortunately, this strategy can damage healthy tissue and has given rise to the description of neutrophils as friend and foe. Despite that, these mechanisms are highly effective against bacteria, fungi and protozoa [3, 4].

A new third line of attack was discovered by Brinkman et al. in 2004 [5]. They observed that neutrophils were capable of releasing their nuclear DNA content in the form of an extracellular fibres, which they aptly named neutrophil extracellular traps (NETs). Since then, there has been a rush of research exploring the mechanism and functioning of NETs, and to a lesser extent extracellular traps generated by other type of cells. This article will review the main outcome of this research, followed by an analysis of how to study the formation of extracellular traps by in vitro isolation and quantification of them.

\section{NETs: what are they}

It should be noted that neutrophils are not the only cells capable of extracellular trap formation. It has been shown that monocytes [6], eosinophils [7] and mast cells [8] are other examples of cells employing this strategy. However, research has focussed on neutrophils and therefore, from all extracellular traps (ETs), neutrophil extracellular traps (NETs) are best understood at this moment.

Brinkman and colleagues [5] described NETs as free structures that are capable of degrading virulence factors, especially trapping and killing bacteria. They found proteins from primary, secondary and tertiary granules as well

Correspondence: Michał Przemysław Pruchniak, Department of Laboratory Diagnostic and Clinical Immunology of Developmental Age, Warsaw Medical University, Marszałkowska 24, 00-576 Warsaw, phone/fax: +48 2262965 17, e-mail: michal.pruchniak@wum.edu.pl 
as histones incorporated in the structure. However, nuclear DNA was found to be the main structural component, with DNAse being capable of degrading the NETs structure. Neutrophil extracellular traps can be formed and released at sites on inflammation as soon as 10 minutes after activation $[5,9]$. This is an active mechanism that is now usually referred to as '(N)ETosis' [5]. Because this strategy kills not only the microorganism, but also the neutrophil itself, it has to be carefully regulated. It has been hypothesized that the neutrophil does this by sequentially using the available strategies, starting with phagocytosis, followed by degranulation and finally, when all else has failed, by NETosis [3]. It is possible for a neutrophil, instead of using nuclear DNA, to form NETs from mitochondrial DNA. This method does not affect the life span of the neutrophil [10]. However, neutrophils seem to rarely employ this method, unlike e.g. eosinophils [3].

In a cell undergoing ETosis, the nucleus loses shape, followed by homogenization of the chromatins. Next, the nuclear envelope breakers, as well as the granular membranes, allowing the chromatins to mix with the granular proteins. Finally the cell membrane breaks allowing the NET to function extracellularly [11]. Pilsczek et al. [9] described an alternative process that seems to coexist with the former. They described a very rapid process, taking 5 to 60 minutes, NETs were formed in part from the nuclear DNA which have been delivered to the cytoplasm by a vesicle originating from the nucleus. As a further step, the nuclear membrane dissipate, releasing more DNA into the cytoplasm, possibly for further NET formation. This vesicle-based NET formation does not seem to occur when mitochondrial DNA is used [9].

The initiation of both forms of NETosis is dependent on the presence of ROS, and a mutation in the gene for NADPH disables NET formation completely [3]. However, at this moment it is unknown how ROS are involved in NET formation. Another factors that is crucial for NET formation is the upregulation of antiapoptotic proteins, thus allowing the cell to undergo NETosis instead of apoptosis. Furthermore, NET formation appears to be dependent on the Raf-MEK-ERK pathway [12].

\section{The functions of NETs}

\section{Bactericidal effects}

As mentioned before, the main function of NETs is to contain and kill invading micro-organisms. These are clearly two distinct functions, because Menegazzi et al. [13] found live bacteria in traps, and several others have described how some bacteria find their ways to escape from the NETs. Menegazzi even hypothesized that NETs have no bactericidal effect, but are only capable of entrapping microorganisms [13]. This, however, is inconsistent with several other studies that did describe a bactericidal properties of NETs. Pilsczek et al. [9] found NETs capable of killing Staphylococcus aureus and Parker et al. [14] described the granular protein myeloperoxidase (MPO), as a part of NET structure, being bactericidal in the presence of hydrogen peroxidase $\left(\mathrm{H}_{2} \mathrm{O}_{2}\right)$, secreted in abundance by neutrophils. Since the presence of ROS is necessary for NET formation, MPO can be considered as bactericidal in all NETs. The abovementioned studies confirm the conclusion that destruction of NETs by DNAse decreased the bactericidal effects of neutrophils [5].

\section{NETs and disease}

The discovery of NETs has had a considerable influence on our understanding of health and disease. For example, it was not understood why statins increase the effectiveness of phagocytes in spite of the fact they decrease ROS bursts and phagocytosis. Recently it has become clear that they enhance NET formation [15]. Another example where NETs seem to play a key role, is sepsis where NET formation is induced by TLR-4-dependent interaction between platelets and neutrophils [16].

Although it has become more and more clear that, like the other measures at the neutrophil's demand, NETs may also have negative impact on human health. For instance Gupta et al. [17] found that NETs formed after activation by placental interleukin 8 (IL-8) and SBTM, plays a pathogenic role in preeclampsia. Moreover Kessenbrock et al. [18] found NETs to trigger vasculitis and to promote the autoimmune response against neutrophil components in patients with small vessel vasculitis.

From a different perspective, Margraf and colleagues [19] found a preliminary evidence that the amount of circulating DNA derived from NETs may become a prognostic marker for posttraumatic development of inflammatory second hit and sepsis. The observation of Lögters et al. [20] in septic arthritis correspond with this findings. Recently different studies started to highlight the role of NETs in different pathological conditions.

\section{Studying NETs}

In order to study the DNA of NETs, or any other type of extracellular trap, three basic steps can be performed. First of all, neutrophils must be isolated from patient blood. Secondly, they must be activated in vitro. Thirdly, the NET can be visualised and/or the ET-DNA can be measured by a quantitative assay of DNA in the sample. To fully evaluated the process of NET formation all three steps should be assessed. However, according to Margraff et al. [19] for the diagnostic purposes the first two steps are redundant.

\section{Isolation and activation of neutrophils}

Isolation of neutrophils can be done easily, either automatically by automatic method or manually with the use of gradients and centrifugation. Always the short life-span of 
neutrophils should be taken into account. Activation can be performed by a multitude of reagents, including bacterial LPS, GM-CSF followed by TLR-4 or C5a [20]. The most frequently used reagent is PMA $[12,14]$. This reagent has a profound effect on the NET formation.

Another factor that influences both the number of viable neutrophils recovered after isolation and their potential to be activated, is the anticoagulant. Freitas et al. [21], observed that EDTA samples gives the highest yield of neutrophils, almost twice higher as heparin samples, which in turn gives a significantly higher yield than citrate. However, heparin and citrate resulted in a higher absolute count of activated cells after in vitro addition of PMA. This suggest that, when neutrophils are isolated with for research purposes that necessitate their in vitro activation, heparin and citrate may be the preferred anticoagulants.

\section{Visualisation of NET}

There are diverse methods for the visualisation of NET. Neutrophil extracellular traps were first discovered using electron microscopy [5]. However, as a quantification technique this would be laborious. Moreover, it can be difficult to distinguish NETs from fibrin fibres [22]. Nonetheless, there are many techniques available based on DNA staining, usually after the application of PCR. These techniques, as well as their advantages and drawbacks will be discussed in the next section.

\section{Quantification of ETs-DNA by fluorescence analysis}

\section{Fluorescence}

The phenomenon of fluorescence was first described by George Stokes in 1852. He noticed that fluorspar and uranium glass have the ability to change invisible light beyond the violet end of the visible spectrum into blue light [23]. Following that discovery fluorometric assays were developed through years. Presently this method is widely used in chemistry, geology, life sciences and forensics.

Fluorescence is used in the life sciences generally as a non-destructive way of tracking or analyzing biological molecules. It was shown that some proteins and small molecules in cells are fluorescent by nature. Phycoerythrin, GFP, chlorophyll and NADH proteins have the ability of autofluorescence (also called intrinsic fluorescence). On the other hand fluorescent dyes can be attached to proteins, nucleic acids, lipids or small molecules. This dye, called fluorophore, can be composed from small molecules, proteins or quantum dots. Fluorophores can be divided into two classes: intrinsic and extrinsic. The intrinsic are those that can be found naturally inside the cell, whereas the extrinsic are artificially added to a specimen that does not display the desired spectral properties [24]. The basic principle is that a fluorophore is a component that causes a molecule to absorb energy of a specific wavelength and then re-emit energy at a different, but equally specific, wavelength. The amount and wavelength of the emitted energy depend on both the fluorophore and its chemical environment [25]. The quantification of a dye is performed with a spectrofluorometer, technique that finds applications in many fields of science such as microscopic observation, immunology, fluorescence-activated cell sorting (FACS), biosensors and genetic material detection.

\section{DNA detection}

The original techniques used for DNA detection were radioactive labelling and silver staining. Both methods had their own limitations and disadvantages. Radioactive labelling visualises components of target molecule with use of $\mathrm{P}^{32}$ that is incorporated into one of the dNTPs. After PCR the DNA strands will contain the radiolabel. When exposed to photographic film the radiation leaves dark bands on the film at the positions of DNA bands in the gel. Major disadvantages of this method are the short half-life of the reagents and the radiation hazards [26]. Alternative method based on silver staining is a quite safe and easy. After DNA amplification and electrophoresis a silver salt solution is added to gel chamber. Silver ions bind to the bases within the gel matrix after which they can be reduced by formaldehyde under alkaline conditions [27]. Silver staining is less hazardous than radioactive detection methods but is not as convenient as fluorescence methods. The primary advantage of silver staining is that reagents are safe and inexpensive. Sensitivity is approximately 100 times higher than that obtained with ethidium bromide staining. The major disadvantages are that image is only of one colour [28].

Current techniques of DNA/RNA labelling are always based on fluorescent dyes. Some dyes can incorporate into DNA during PCR, others like SYBR Green intercalate into DNA structure. The mechanism can be based on the introduction of aldehyde groups by partial depurination of the DNA or oxidation of the 3'-terminal ribonucleoside in RNA by sodium periodate. Fluorescent labels with an attached hydrazine group are efficiently coupled with the aldehyde groups and the hydrazone bonds are stabilized by reduction with sodium cyanoborohydride. Alternatively, DNA can be quantitatively split at the depurinated sites with ethylenediamine. The aldimine bond between the aldehyde group in depurinated DNA or oxidized RNA and ethylenediamine is stabilized by reduction with sodium cyanoborohydride and the primary amine group introduced at these sites is used for attachment of isothiocyanate or succinimide derivatives of fluorescent dyes. The fluorescent DNA labelling can be carried out either in solution or on a reverse phase column [29]. These procedures are very convenient because they are safe and they can incorporate one fluorescent dye molecule per DNA/RNA fragment, thus quantitative measurements can be performed. Moreover more than single 
colour of dye can be used allowing distinguish one kind of DNA from another and multiplex polymerase chain reaction can be carry out as well $[29,30]$.

\section{Attempts of extracellular traps-DNA quantification based on fluorescence}

In most studies ETs were derived from neutrophils. Neutrophil extracellular traps generated by activated neutrophils are quite difficult for quantitative analysis. The DNA backbone of this structure is filled with variety of cell proteins. It also seems that these structures are connected with cell fragments. Fuchs and colleagues showed that NETs can only form when the cell membrane was ruptured in a way that differ from apoptosis. They used an Annexin V test that clearly demonstrates that NETs emerge from dying neutrophils. This also shows that NETs are not released by apoptotic neutrophils because phosphatidylserine was not exposed before the rupture of the plasma membrane, as indicated by the loss of calcein blue [31].

Due to the fact that DNA is a major structural component of NETs, the fluorescent DNA stains Sytox ${ }^{\circledR}$ Green and Sytox ${ }^{\circledR}$ Orange are often used to both visualize NET release from neutrophils and to quantify fluorometric NETsDNA [10, 32-34 and others]. Both Sytox ${ }^{\circledR}$ dyes have a high affinity for nucleic acids. They only penetrate cells with disrupted plasma membranes because they cannot cross the membranes of live cells of the choice between both dyes is determined by filter of a fluoroscope. While Sytox ${ }^{\circledR}$ Green gives a bright green picture when excited with the 450-490 nm laser, Sytox ${ }^{\circledR}$ Orange is excited by $520-550 \mathrm{~nm}$ source and gives a bright orange light $[35,36]$. Picogreen ${ }^{\circledR}$ and Hoechst 33258 - based assay are also used to check the NETs-DNA [31, 33, 37, 39]. Both dyes are ultra sensitive fluorescent nucleic acid stains for quantifying double-stranded DNA in solution. The first gives a bright green fluorescence whereas another is a blue dye. It seems that Picogreen ${ }^{\circledR}$ staining is better for NETs quantitative analysis due to the fact that Hoechst-based assay is selective for dsDNA and does not show significant fluorescence enhancement in the presence of proteins which can be found within NETs structure [37-39].

Assays are often performed in 96-well microplates. Sytox ${ }^{\circledR}$ Dyes are also bound to cell membrane fragments, thus the sample after incubation and induction of NETs cannot be measured without further maintenance. This maintenance includes the use of nuclease enzyme that is necessary to detach ETs from cell fragments which sediment in the sample [40]. The bacterial nuclease or MNase, which induces partial digestion, is widely used, though its concentration and reaction time differ within protocols. After incubation with such enzymes a chelating agents must be used in order to stop DNA fragmentation. Usually EDTA is sufficient but ethylene glycol tetraacetic acid (EGTA) can be used when calcium ions are investigated as well [32, 37]. After partial digestion the well-plates are centrifuged. This step cause DNA fragmentation in wells. The pellet contains all cell fragments and unnecessary proteins whereas the supernatant contains the soluble ETs. Another advantage is that the quantitative fluorescence assays does not have to be performed immediately, because supernatants samples after nuclease treatment can be stored at $4^{\circ} \mathrm{C}$ until further use $[31,34]$. Since only a certain percentage of cells in a culture produce ETs, it is good to estimate the percentage of released ET-DNA. In order to do that it is necessary to isolate whole genomic genetic material from the same number of cells which are used for ETs generation. The percentage of cell undergoing ETosis can be calculated by dividing the amount of isolated ET-DNA through the average genomic DNA content [31].

\section{Summary}

During the past few years ETs were studied very intensively. However, the methods by which ETs are explored still largely differ between studies and an optimal, standardized procedure is still lacking, though it could significantly aid in the development of our understanding of ETs. However, fluorescent staining with the purpose of quantitative analysis of ET-DNA seems promising to be feasible and useful, both for clinical studies and diagnostic purposes.

\section{References}

1. Borregaard N (2010): Neutrophils, from marrow to microbes. Immunity 30: 657-670.

2. Soehnlein O (2009): An elegant defense: how neutrophils shape the immune response. Trends Immunol 30: 511-512.

3. Papayannopoulos V, Zychlinsky A (2009): NETs: a new strategy for using old weapons. Trends Immunol 30: 513-521.

4. Polańska B (2009): Chronic granulomatous disease - primary phagocytic immunodeficiency. Centr Eur J Immunol 34: 182-191.

5. Brinkmann V, Reichard U, Goosmann C, et al. (2004): Neutrophil extracellular traps kill bacteria. Science 303: 15321535.

6. Webster SJ, Daigneault M, Bewley MA, et al. (2010): Distinct cell death programs in monocytes regulate innate responses following challenge with common causes of invasive bacterial disease. J Immunol 185: 2968-2979.

7. Simon D, Hoesli S, Roth N, et al. (2011): Eosinophil extracellular DNA traps in skin diseases. J Allergy Clin Immunol 127: 194-199.

8. von Köckritz-Blickwede M, Goldmann O, Thulin P, et al. (2008): Phagocytosis-independent antimicrobial activity of mast cells by means of extracellular trap formation. Blood 111: 3070-3080.

9. Pilsczek FH, Salina D, Poon KK, et al. (2010): A novel mechanism of rapid nuclear neutrophil extracellular trap formation in response to Staphylococcus aureus. J Immunol 185: 74137425.

10. Yousefi S, Mihalache C, Kozlowski E, et al. (2009): Viable neutrophils release mitochondrial DNA to form neutrophil extracellular traps. Cell Death Differ 16: 1438-1444. 
11. Brinkmann V, Laube B, Abu Abed U, et al. (2010): Neutrophil extracellular traps: how to generate and visualize them. J Vis Exp 36: e1724.

12. Hakkim A, Fuchs TA, Martinez NE, et al. (2011): Activation of the Raf-MEK-ERK pathway is required for neutrophil extracellular trap formation. Nature Chem Biol 7: 75-77.

13. Menegazzi R, Decleva E, Dri P (2012): Killing by neutrophil extracellular traps: fact or folklore. Blood 119: 1214-1216.

14. Parker H, Albrett AM, Kettle AJ, Winterbourn CC (2012): Myeloperoxidase associated with neutrophil extracellular traps is active and mediates bactericidal killing in the presence of hydrogen peroxide. J Leukoc Biol 91: 369-376.

15. Chow OA, von Köckritz-Blickwede M, Bright AT, et al. (2010): Statins enhance formation of phagocyte extracellular traps. Cell Host Microbe 8: 445-454.

16. Clark SR, Ma AC, Tavener SA, et al. (2007): Platelet TLR4 activates neutrophil extracellular traps to ensnare bacteria in septic blood. Nat Med 13: 463-469.

17. Gupta AK, Hasler P, Holzgreve W, et al. (2005): Induction of neutrophil extracellular DNA lattices by placental microparticles and IL-8 and their presence in preeclampsia. Hum Immunol 66: 1146-1154.

18. Kessenbrock K, Krumbholz M, Schönermarck U, et al. (2009): Netting neutrophils in autoimmune small-vessel vasculitis. Nat Med 15: 623-625.

19. Margraf S, Lögters T, Reipen J, et al. (2008): Neutrophilderived circulating free DNA (CF-DNA/NETs): a potential prognostic marker for posttraumatic development of inflammatory second hit and sepsis. Shock 30: 352-358.

20. Lögters T, Paunel-Görgülü A, Zilkens C, et al. (2009): Diagnostic accuracy of neutrophil-derived circulating free DNA for septic arthritis. J Orthop Res 6: 1401-1407.

21. Freitas M, Porto G, Lima JL, Fernandes E (2008): Isolation and activation of human neutrophils in vitro. The importance of the anticoagulant used during blood collection. Clin Biochem 41: 570-575.

22. Krautgartner WD, Klappacher M, Hannig M, et al. (2010): Fibrin mimics neutrophil extracellular traps in SEM. Ultrastruct Pathol 34: 226-231.

23. Stokes GG (1852): On the change of refrangibility of light. Philos Trans of the R Soc Lond 142: 463-562.

24. Lakowicz JR (2006): Principles of fluorescence spectroscopy. $3^{\text {rd }}$ ed. Springer, 2006.

25. Sauer M, Hofkens J, Enderlein J (2011): Handbook of fluorescence spectroscopy and imaging. Wiley- $\mathrm{VCH}$ Verlag $\mathrm{GmbH}$ \& Co. KGaA, Weinheim 2011.

26. Carpenter WC, Rai DV, Samuel AR, Höfner MC (1996): Comparison of a radioactive and non-radioactive method for sequencing foot and mouth disease virus isolates. Rev Sci Tech 15: 875-882.

27. Byun SO, Fang Q, Zhou H, Hickford JG (2009): An effective method for silver-staining DNA in large numbers of polyacrylamide gels. Anal Biochem 385: 174-175.

28. Butler JM. Forensic DNA typing: biology, technology, and genetics of STR markers. Elsevier, USA 2005.

29. Proudnikov D, Mirzabekov A (1996): Chemical methods of DNA and RNA fluorescent labeling. Nucleic Acids Res 24: 4535-4542.

30. Hayden MJ, Nguyen TM, Waterman A, Chalmers KJ (2008): Multiplex-ready PCR: a new method for multiplexed SSR and SNP genotyping. BMC Genomics 9: 80.
31. Fuchs TA, Abed U, Goosmann C, et al. (2007): Novel cell death program leads to neutrophil extracellular traps. J Cell Biol 176: 231-241.

32. Leffler J, Martin M, Gullstrand B, et al. (2012): Neutrophil extracellular traps that are not degraded in systemic lupus erythematosus activate complement exacerbating the disease. J Immunol 188: 3522-3531.

33. Yan J, Meng X, Wancket LM, et al. (2012): Glutathione reductase facilitates host defense by sustaining phagocytic oxidative burst and promoting the development of neutrophil extracellular traps. J Immunol 188: 2316-2327.

34. Palmer LJ, Cooper PR, Ling MR, et al. (2012): Hypochlorous acid regulates neutrophil extracellular trap release in humans. Clin Exp Immunol 167: 261-268.

35. Invitrogen: SYTOX ${ }^{\circledR}$ Green Nucleic Acid Stain. http://tools. invitrogen.com/content/sfs/manuals/mp07020.pdf

36. Invitrogen: SYTOX ${ }^{\circledR}$ Orange Nucleic Acid Stain. http://probes. invitrogen.com/media/pis/mp11368.pdf

37. Saffarzadeh M, Juenemann C, Queisser MA, et al. (2012): Neutrophil extracellular traps directly induce epithelial and endothelial cell death: a predominant role of histones. PLoS One 7: e32366.

38. Invitrogen: Quant-iT ${ }^{\mathrm{TM}}$ PicoGreen ${ }^{\circledR}$ dsDNA Reagent and Kits. http://probes.invitrogen.com/media/pis/mp07581.pdf

39. nvitrogen: Hoechst Stains. http://probes.invitrogen.com/ media/pis/mp21486.pdf

40. Pruchniak MP, Araźna M, Demkow U (2012): Extracellular traps formation and visualization methods. Centr Eur J Immunol 37: 81-84. 